\title{
Prediction of the Bandgap of a Core-Shell Microsphere via Light Intensity Fluctuations
}

\author{
Moon Kyu Choi and Youngjin Choi \\ Department of Chemical Engineering, Hongik University, Mapo-Gu, Seoul 121-791, Republic of Korea \\ Correspondence should be addressed to Moon Kyu Choi, moonkyu@hongik.ac.kr
}

Received 27 July 2010; Accepted 16 September 2010

Academic Editor: Canan Varlikli

Copyright (๑) 2011 M. K. Choi and Y. Choi. This is an open access article distributed under the Creative Commons Attribution License, which permits unrestricted use, distribution, and reproduction in any medium, provided the original work is properly cited.

It has been experimentally observed that in the case of microspheres irradiated by light, the absorption wavelength shift occurs, known as the blueshift, with changing shell materials (i.e., by decreasing the refractive index of the shell). In the present investigation, we want to demonstrate it numerically by using the boundary element method. The material used for the simulation is a core-shell $\left(\mathrm{SiO}_{2}\right.$ and another material of a larger refractive index) microsphere and it is irradiated by unpolarized monochromatic light wave. This paper intends to demonstrate that it is possible to predict the bandgap of a core-shell microsphere resulting from two different bandgap materials and that the numerical simulation employed produces the blueshift.

\section{Introduction}

There are many different methods to produce semiconductor nanocrystals and consequently they will have different optical and structural properties depending on how they are produced. Above all, one is interested in the bandgap of the newly synthesized material because it is directly related to light absorption and luminescence wavelength. Primarily of interest is the realization of the wavelength range for nanocrystal-based green LEDs and white-light generation. One of the exciting examples is $\mathrm{Cd}_{1-x} \mathrm{Zn}_{x} \mathrm{Se}$ alloy nanocrystals because the luminescence wavelength from them may be tuned by changing the composition $(\mathrm{Cd}: \mathrm{Zn})$ without changing the nanocrystal size $[1,2]$. Depending on the production methods, the filmed materials will have different optical and structural properties including bandgaps. One of the most desirable abilities that we want to have regarding the design of thin film electronic devices is to predict the bandgap magnitude of the coated devices, given the bandgaps of each material.

A key factor for the development of photonic integrated circuits (PICs) is its ability to selectively control the bandgap across a semiconductor wafer. Since the semiconductor bandgap can be easily controlled during the growth, the use of selective epitaxial growth, or etching and regrowth, has been extensively studied for different semiconductor platforms. Quantum well intermixing (QWI) is a wellknown method for the postgrowth modification in QW heterostructures eliminating the need for epitaxial regrowth.

A phenomenon showing light-matter interaction is the photonic confinement in a small dimension which sets the vacuum electric field amplitude of the cavity photons. Another way to explain this phenomenon is that one can interpret the intensity fluctuations as the cause of energy conversion according to the fluctuation-dissipation theorem. Quantum effects have been investigated recently to show chaos-like properties (i.e., noise or fluctuations) on a single barrier potential and on a one-dimensional periodic potential barrier. The surface covered with crystallites may be modeled with those periodic potentials. The researchers used either the plane wave of light or a wavepacket (pulse) passing through the potential barrier. They demonstrated that the chaos was created by the bounded one-dimensional multibarrier potential $[3,4]$ and proved it is an ordered and periodic phenomenon. Also another group demonstrated unexpected behavior of a dissipative particle in simple multiscale systems subject to chaotic noise and clarified that the reason for the particular behavior was because it is under a periodic potential [5]. They concluded that the occurrence of drift in a symmetric periodic potential would be expected 
to be a common feature of noise-driven systems. Essentially, the microsphere irradiated by light is quite similar to a particle placed in a potential well of finite barrier [6] since what we are trying to find is the photon distribution on the crystallite surface.

Our goal is that we can predict the bandgap of a thinfilmed material once we know the bandgaps of the composing materials. To this end in this paper, we want to demonstrate how to predict the location of absorption wavelengths for a core-shell microsphere, depending on the shell material. One of the best ways to uphold the present numerical method would be to demonstrate the blueshift phenomenon observed widely experimentally. One of the authors has been studying this problem recently, publishing a few papers [7-9], and this paper may be taken as an extension of these consecutive efforts. Especially, a recent publication [10] predicting the blueshift with respect to the shell layer thickness has been accomplished and the author is trying to deepen the relevant theory in this paper.

\section{Theoretical Background}

We are considering here a core-shell type microsphere irradiated by monochromatic unpolarized light wave and investigating the realization of light intensity fluctuations at the absorption wavelengths. By finding the wavelength yielding the most significant fluctuations, one can estimate the bandgap of the thin-filmed material. In a recent publication [10], the author demonstrated the blueshift of the absorption wavelength due to the decrease in the shell thickness. We want to extend this analysis to find the effect of the refractive index of the shell material on the blueshift.

Figure 1 displays a schematic of the present situation. The increase of the refractive index of the shell signifies that the quantum well deepens more. Contrarily, if one reduces the refractive index of the shell, the depth of the quantum well becomes shallow (Refer to Figure 2). In relation to this change of shell material, we want to show numerically the blueshift phenomenon which was observed experimentally by other researchers. This is the phenomenon that the absorption (or the luminescence) wavelength decreases with decreasing refractive indices of the shell [11]. Since this investigation is an extension of the former ones, most mathematical formulations are omitted here and only essential contents are stated. The details may be looked up in the previous publications [7-9].

Readers are especially advised to see Reference [12] for the modeling details. The electric and magnetic vectors in both the internal and the external regions must satisfy the macroscopic Maxwell equations that govern the behavior of electromagnetic fields. Taking the harmonic time dependence to be $\exp (-\iota \omega t)$ for all fields and assuming no free charge, we reduce the Maxwell equations to a set of vector wave equations for the electric field $\mathbf{E}$ and the magnetic field H:

$$
\begin{gathered}
\nabla^{2} \mathbf{E}+k^{2} \mathbf{E}=0, \\
\nabla^{2} \mathbf{H}+k^{2} \mathbf{H}=0,
\end{gathered}
$$

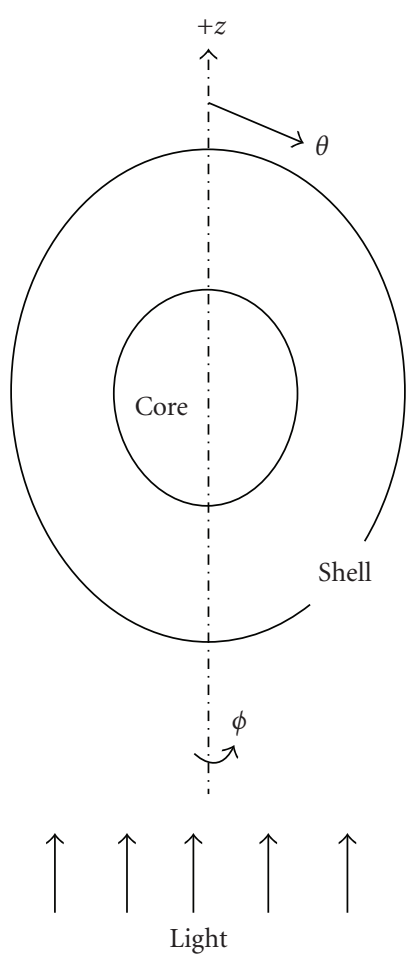

Figure 1: Schematic diagram of a microsphere. The monochromatic unpolarized light irradiates a core-shell microsphere in the $+z$ direction.

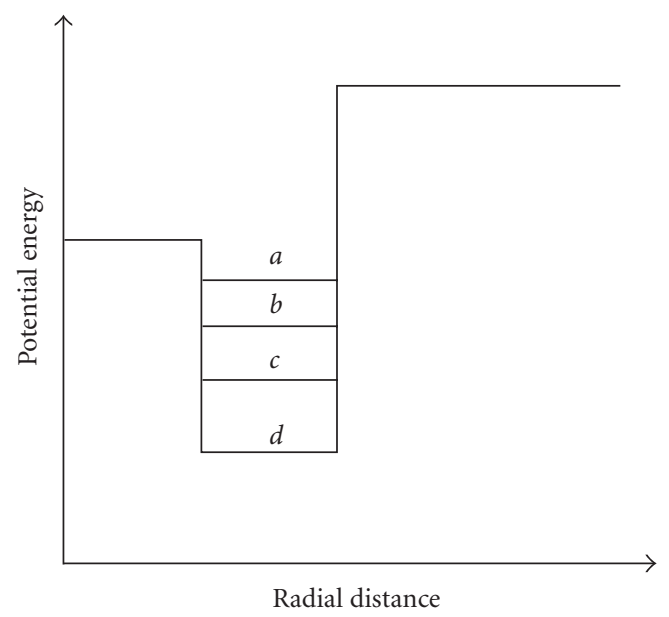

Figure 2: Potential energy variation along the radius for a coreshell microsphere. This figure shows the meaning of the varying refractive index of the shell. The higher the refractive index (n) of the shell, the deeper the quantum well. $n(a)<n(b)<n(c)<n(d)$.

where $k$ is the wave number, $k=\omega(\epsilon \mu)^{1 / 2}, \epsilon$ is the complex permittivity, and $\mu$ is the permeability.

It is very difficult to solve (1) and (2) numerically. There are three scalar equations coupled to solve simultaneously for each layer, since (1) and (2) are separate vector equations. To treat the present case, one has to solve nine equations in general simultaneously. One can derive, however, the governing equations for the Debye potentials, which are 
TABLE 1: Parameters for modeling and resulting absorption wavelengths.

\begin{tabular}{lccccccc}
\hline Case & $\# 1$ & $\# 2$ & $\# 3$ & $\# 4$ & $\# 5$ & $\# 6$ & $\# 7$ \\
\hline Refractive index (core) & $1.48+\mathrm{i} 0.01$ & $1.48+\mathrm{i} 0.01$ & $1.48+\mathrm{i} 0.01$ & $1.48+\mathrm{i} 0.01$ & $1.48+\mathrm{i} 0.01$ & $1.48+\mathrm{i} 0.01$ & $1.48+\mathrm{i} 0.01$ \\
Refractive index (shell) & $1.60+\mathrm{i} 0.01$ & $1.80+\mathrm{i} 0.01$ & $2.00+\mathrm{i} 0.01$ & $2.20+\mathrm{i} 0.01$ & $2.40+\mathrm{i} 0.01$ & $2.60+\mathrm{i} 0.01$ & $2.80+\mathrm{i} 0.01$ \\
representative wavelength $[\mathrm{nm}]$ & 450 & 470 & 490 & 500 & 540 & 540 & 570 \\
Bandgap of shell $[\mathrm{eV}]$ & 2.76 & 2.64 & 2.54 & 2.48 & 2.30 & 2.30 & 2.18 \\
\hline
\end{tabular}

equivalent to Scrödinger equations, to represent the TM and TE modes from (1) and (2) as done in the Mie method of solution. For the particle or the air outside the particle, the Debye potentials are governed by

$$
\nabla^{2} u+k^{2} u=0
$$

Note that the scalar governing equations for each layer are separate and independent of each other; this is the great advantage of employing the Debye potentials over solving (1) and (2) directly.

One has to note that these transformed equations are, as a matter of fact, Schrödinger equations which govern the quantum behavior of particles, or photons in this case. The wave number, $k$, is related to the refractive index of particle or medium, $N$ as follows:

$$
k=\frac{N \omega}{c} .
$$

Here the frequency, $\omega$, and the light speed in vacuum, $c$, are constant, but the refractive index changes depending on particle material. We know from the quantum mechanics that the wave number is also related to the potential energy $(U)$ as

$$
k^{2}=(E-U) \frac{8 \pi^{2} m}{h^{2}} .
$$

The significance of bigger wave number is lower potential energy. In this study, we want to treat a double-layer microsphere with crystalline unit cells covered on, so photons should experience different potential energies depending on whether they are placed on the edges of the cells or their inside.

The boundary element method developed recently by the author in a previous investigation [8] is extended to study an absorption problem on a microsphere and suggests a method to predict the light intensities on $\mathrm{TiO}_{2}$ microsphere. Since the governing equations in the present investigation, the Helmholtz-type equations, admit of the boundary-element formulation, one needs only calculate two integral equations simultaneously for each surface. When we want to deal with a single-layer microsphere, however, we need to make two layers in a coated microsphere be of the same material.

There are many factors that influence light intensity fluctuations. They are, to name a few, the crystalline size, the shell layer (or film) thickness, the particle size, and so forth. Besides other chemical and physical properties including chemical compositions, passivation and surface morphology may also be in effect. We need to demonstrate blueshift theoretically (i.e., numerically) somehow with those causes. In this paper we, especially want to study on the effects of the shell material (or its refractive index) on the blueshift, fixing other parameters. The realization of the blueshift by the boundary element method would be another great proof for the author's recent successive publications.

The properties of any thermodynamic system in macroscopic equilibrium with its surroundings, for instance, the energy, and the density, undergo microscopic fluctuations, but give us only average values. The intrinsic fluctuations in matter-density are most easily observed by the scattering of light which they produce in transparent systems. Another well-known example is the critical opalescence with the substance at the critical point. The fluctuation-dissipation theorem [13] expresses a relation between the susceptibility of an irreversible response and equilibrium fluctuations. Phenomenologically it is found that the flow of energy in a thermally conducting body is proportional to the gradient of the temperature. Likewise the electric potential difference causes the flow of electrons in a conductor. The phenomenological thermal and electrical conductivities are related to the autocorrelations of fluctuations of some properties. Similarly, the energy conversion from one type to another may occur from the fluctuations of energy on the interface. This research area is named as fluctuation-induced transport [5].

The problem considered in the present investigation is about the steady-state photon densities inside a microscale double-layer dielectric sphere, given the surface intensities of the incident light. The sphere may be, therefore, considered as a thermodynamic equilibrium system and the fluctuations of light intensities should be related to some kind of energy conversion rates by the fluctuation-dissipation theorem. The electron transfer among microstates can happen after part of input photon energy is transferred to the particle surface. These excited electrons may contribute to photovoltaic effects, photocatalytic behaviors, and photoluminescence.

\section{Results and Discussion}

One of the main purposes of this paper is to demonstrate the blueshift numerically. For this purpose Table 1 and the following set of parameters are employed for typical demonstrations. We studied seven different shell materials in the present investigation, starting with the smallest refractive index (or the highest or most shallow well according to Figure 2) and then increasing the refractive index little by little. We found the representative absorption wavelength around which significant fluctuations were seen. 


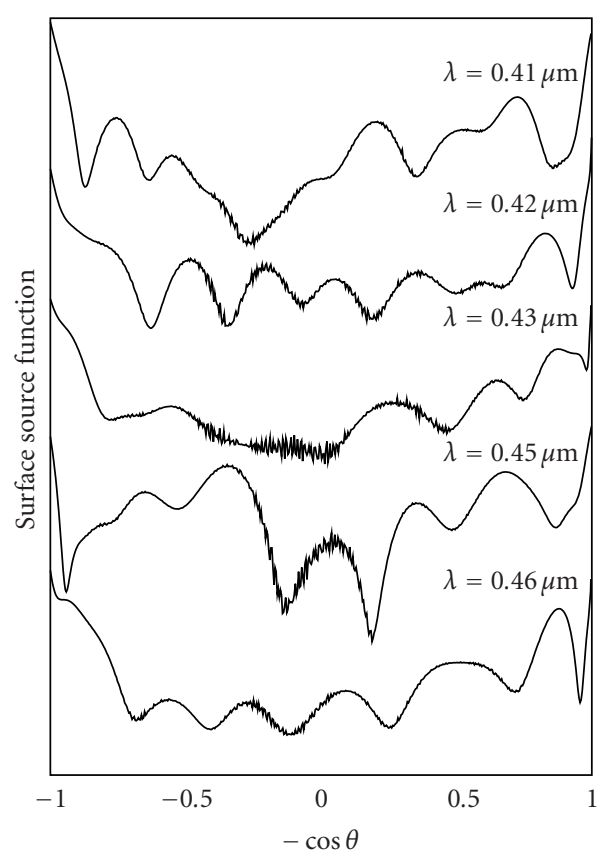

(a)

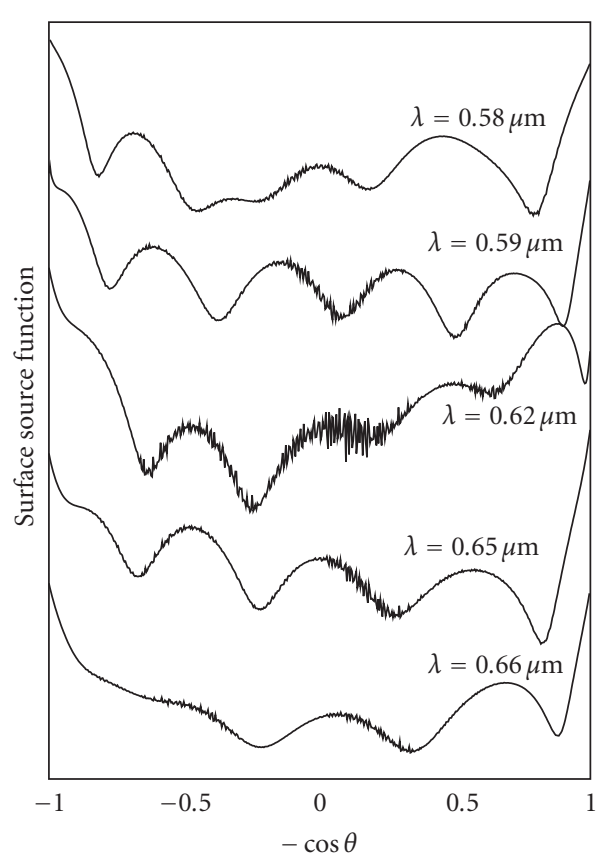

(b)

Figure 3: Source functions versus cos(angle) for a single-layer microsphere of $500 \mathrm{~nm}$ radius, the crystal size of $3.0 \mathrm{~nm}$, and many different wavelengths. (a) For the wavelengths from $410 \mathrm{~nm}$ to $460 \mathrm{~nm}$. (b) For the wavelengths from $580 \mathrm{~nm}$ to $660 \mathrm{~nm}$. Other wavelengths between 100 and $1000 \mathrm{~nm}$ were treated, but they are not shown here because they do not produce fluctuations. The number of surface peripheral elements was 525 . The refractive index on the unit cell edge was chosen to be $2.80+\mathrm{i} 0.01$. The abscissa tic -1 corresponds to a point on the particle surface opposite the incident light (in the forward scattering direction) and +1 corresponds to the point nearest the incident light source (in the back scattering direction).

For one thing, one needs to find the relationship of the bandgap of a homogeneous microsphere with its refractive index. For this purpose we, selected various refractive indices along with a fixed set of other parameters including the microsphere size and the crystallite size. With each refractive index, we first calculated the surface intensities and found the locations of fluctuations. Figure 3 displays to explain how we find the locations of fluctuations. The angle in the $x$-axis of graphs is the azimuthal angle measured from the $+z$-axis, the light propagation direction as shown in Figure 1. The value of +1 implies the peripheral location where the sphere is exposed to the incident light whereas -1 is the opposite location. The source functions are the ratio of the electric field magnitude of the incident light to that of the absorption light. One may regard this source function as the intensity ratio.

(i) light: $100-1100 \mathrm{~nm}$ wavelength at $10 \mathrm{~nm}$ intervals and unpolarized,

(ii) microparticle radius: $500 \mathrm{~nm}$,

(iii) core: $\mathrm{SiO}_{2}$,

(iv) the refractive index of core: $1.48+\mathrm{i} 0.01$,

(v) shell: various materials with different refractive indices,

(vi) medium outside the microsphere: air,

(vii) incident direction of light: $+z$-axis.
The numerical results obtained using these data are shown in Figures 3-5. We calculated the surface intensity with so many different refractive indices and the crystallite size of $3.0 \mathrm{~nm}$ when it is a homogeneous microsphere. Only some of them, especially the ones giving distinctive fluctuations, are displayed in Figure 3. Figure 3 shows only one case of a homogeneous microsphere, using 10 different wavelengths which generate fluctuations, for the refractive index of $2.8+\mathrm{i} 0.01$ (i.e., $\mathrm{TiO}_{2}$ ). From Figure 3(a), one can see that there is a minor range of absorption wavelength between 410 and $460 \mathrm{~nm}$, and Figure 3(b) yields a major range between 580 and $660 \mathrm{~nm}$ with the representative wavelength of $620 \mathrm{~nm}$. Many other wavelengths were successfully treated, but they were not displayed because of no remarkable fluctuations of light intensity on the particle surface. There are two ranges of wavelength, major and minor, where one can see intensity fluctuations. We think that the major fluctuations are related to the bandgap of the material. The wavelength yielding the strongest fluctuations is termed as the representative wavelength for light absorption. The details of how to find such locations of absorption wavelength may be looked up in the references published formerly by one of the authors.

The graphs similar to Figure 3 regarding how to determine absorption wavelengths were obtained with many refractive indices of the shell covering from 1.6 to 2.8 , but they are left out of display. Instead we present the 


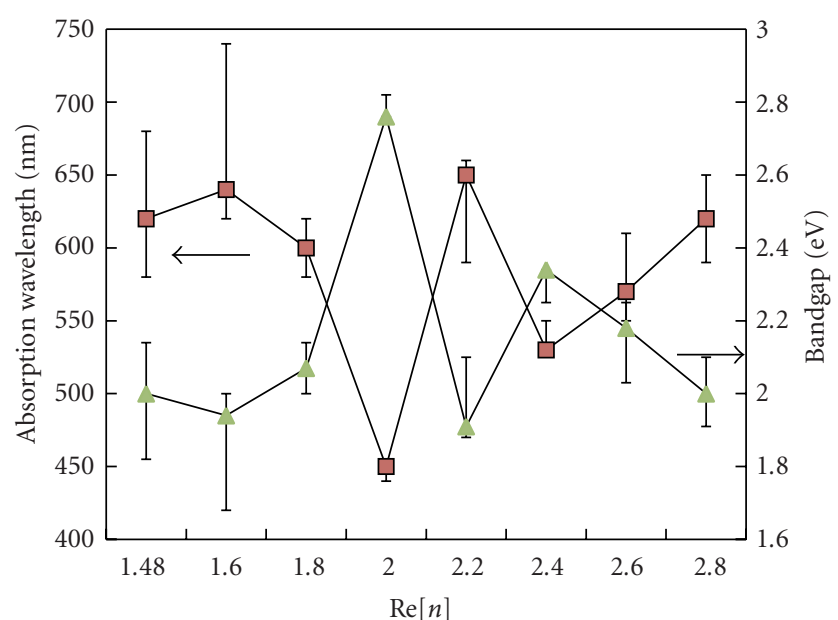

Figure 4: The absorption wavelengths and bandgaps versus the real part of the refractive index are summarized here after their identifications from the figures such as Figure 3 for a homogeneous microsphere. The points are the exact locations of wavelength for the strongest fluctuations while the vertical bars represent a range where fluctuations are seen.

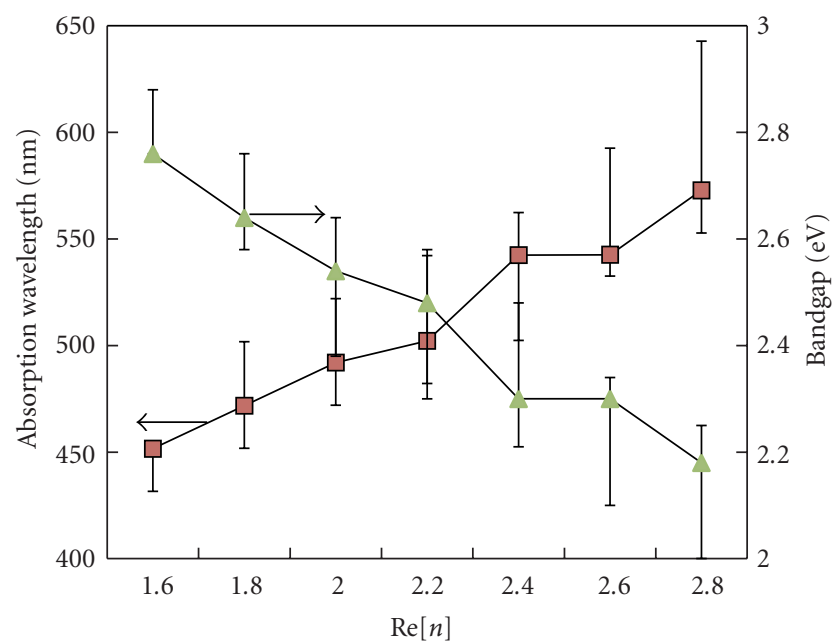

Figure 5: The absorption wavelengths and bandgaps versus the real part of the refractive index are summarized here after their identifications from the figures such as Figure 3 for a core-shell microsphere. The points are the exact locations of wavelength for the strongest fluctuations while the vertical bars represent a range where fluctuations are seen.

locations of the representative wavelength and the major wavelength range in Figure 4. Furthermore, the wavelength can be converted to the bandgap energy $[\mathrm{eV}]$ and shown in Figure 4. One can see that the bandgap energy does not show any tendency with the refractive index of the material. Consequently, it is not possible to predict the bandgap of a homogeneous material when we have the refractive index data. In the same way as with Figure 3, we found all the resulting graphs showing fluctuations and found major ranges of absorption wavelengths and representative wavelengths, showing the absorption wavelengths and their corresponding bandgaps in Figure 4. One can see no correlations between the refractive index of the material and the absorption wavelength (or the bandgap) from Figure 4. This is because a homogeneous microsphere is not a quantum well unlike a core-shell. Even so it is remarkable that one cannot predict any relationship between them. The authors tried to find any relating experimental proofs to support this unusual phenomenon obtained numerically, but it seems that there are no such reports. The authors are guessing that previous experiments done on homogeneous microspheres, if any, with various refractive indices produced only scattered data and that the researchers gave up their publications.

Now we want to present the results and discussion for core-shell microspheres made by coating a shell material on a core. The materials are identified by their refractive indices. In this investigation, $\mathrm{SiO}_{2}$ is chosen to be the core material with its refractive index of $1.48+\mathrm{i} 0.01$. With the refractive index $(1.60+\mathrm{i} 0.01)$ for the shell material, we carried out the calculation of the surface intensity on the core-shell microsphere and found the location of the light wavelength yielding the most severe fluctuations. Then we increased the refractive index little by little, continuing the same calculations. How to find the representative wavelengths and the wavelength ranges of fluctuations is the same as explained above with homogeneous microspheres. They are displayed in Figure 5, showing the absorption wavelength and bandgap energy versus the shell refractive index.

Figure 5 shows representative wavelengths versus refractive indices while the errorbar means the range where significant fluctuations were found and one can see the blueshift of the absorption wavelength as the refractive index of the shell decreases or as the quantum well deepens less. By looking up this figure we may obtain the appropriate refractive index corresponding to a certain bandgap. For instance, if one has a certain material with known bandgap, then he or she can find the corresponding refractive index from Figure 5. When we want to make a new thin-filmed material, we are curious about the bandgap it will have and this is what this research mainly focuses on. Especially, when we want to take advantage of the quantum well intermixing by using the annealing, we need to know beforehand the information of the refractive index versus the bandgap.

The method of quantum well mixing changes surface compositions, and consequently one can generate many different refractive indices using annealing. There are many experimental researches showing the dependence of the blueshift on the compositional variation, but we could find only a single group whose study may be compared with ours because their results are presented in terms of refractive indices. The research group yielded films with refractive indices ranging from 1.5 to $1.85[14,15]$. Then they performed experiments to find emission wavelengths, showing the blueshift. However, their results show a maximum of net blueshift and this is not consistent with the present result where the blueshift is monotonous with the size of the refractive index. The same group performed the thermal annealing treatments on dielectric films and then showed the net blueshift versus film refractive index, which showed also a maximum with respect to the refractive 
index. These two results are not reliable and not agreeing with the present numerical results. It seems they did not single out one parameter like the refractive index, keeping others constant, and thus their results got messed up. However, they did similar research $[16,17]$ later and yielded monotonous blueshifts which are consistent with the present numerical results. On the other hand, two other groups had done similar experiments and found that photoluminescence (PL) emission showed the blueshift monotonously with the annealing temperature $[18,19]$. Annealing must change the composition of the surface and the refractive index, so one may guess that the PL emission should show the blueshift with the decreasing refractive index and their consequences are really consistent with the present numerical results. They showed the annealing treatment changed both the surface compositions and the quantum well thickness. Probably, it should produce different refractive indices derived from transformed surface compositions.

\section{Conclusions}

A core-shell microsphere with the core of $\mathrm{SiO}_{2}$ is investigated with various shell materials of different refractive indices. Since the shell layer functions as a quantum well, varying the refractive index of the shell means changing the depth of the quantum well. We searched the absorption wavelength corresponding to the strongest intensity fluctuations and demonstrated the blueshift of the wavelength as the refractive index of the shell decreased (or quantum well becomes shallower). This demonstration is entirely agreeing with the experiments from other research groups.

\section{Acknowlegments}

This work was supported by the 2009 Hongik University Academic Research Support Fund.

\section{References}

[1] X. Zhong, M. Han, Z. Dong, T. J. White, and W. Knoll, "Composition-tunable $\mathrm{Zn}_{\mathrm{x}} \mathrm{Cd}_{1-\mathrm{x}} \mathrm{Se}$ nanocrystals with high luminescence and stability," Journal of the American Chemical Society, vol. 125, no. 28, pp. 8589-8594, 2003.

[2] X. Zhong, S. Liu, Z. Zhang, L. Li, Z. Wei, and W. Knoll, "Synthesis of high-quality CdS, $\mathrm{ZnS} 5$ and $\mathrm{Zn}_{\mathrm{x}} \mathrm{Cd}_{1-\mathrm{x}} \mathrm{S}$ nanocrystals using metal salts and elemental sulfur," Journal of Materials Chemistry, vol. 14, no. 18, pp. 2790-2794, 2004.

[3] D. Bar and L. P. Horwitz, "Dynamical effects of a onedimensional multibarrier potential of finite range," European Physical Journal B, vol. 25, no. 4, pp. 505-518, 2002.

[4] T. Hondou and Y. Sawada, "Dynamical behavior of a dissipative particle in a periodic potential subject to chaotic noise: retrieval of chaotic determinism with broken parity," Physical Review Letters, vol. 75, no. 18, pp. 3269-3272, 1995.

[5] D. R. Chialvo, M. I. Dykman, and M. M. Millonas, "Fluctuation-Induced transport in a periodic potential: noise versus chaos," Physics Review Letters, vol. 78, no. 8, p. 1605, 1997.

[6] C. Cohen-Tannoudji, B. Diu, and F. Laloë, Quantum Mechanics, Wiley, New York, NY, USA, 1977.
[7] M. K. Choi and J. Kim, "Light intensity fluctuations on a semiconductor microsphere calculated by boundary element method," Current Applied Physics, vol. 7, no. 6, pp. 622-628, 2007.

[8] M. K. Choi, "Light intensity fluctuations on a layered microsphere irradiated by a monochromatic light wave: modeling of an inhomogeneous cellular surface with numerical elements," Materials Science and Engineering B, vol. 137, no. 1-3, pp. 138143, 2007.

[9] M. K. Choi and J. Pyun, "Light intensity and its fluctuations on a layered microsphere: Effects of shell thickness acting as a quantum well," Current Applied Physics, vol. 8, no. 5, pp. 603$611,2008$.

[10] M. K. Choi and Y. Choi, "Numerical demonstration of the blueshift of the light absorption wavelength for a layered microsphere: effects of shell thickness on the blueshift," Journal of the Korean Physical Society, vol. 54, no. 6, pp. 23092317, 2009.

[11] T. Y. Lin, "Converse piezoelectric effect and photoelastic effect in InGaN/GaN multiple quantum wells," Applied Physics Letters, vol. 82, no. 6, pp. 880-882, 2003.

[12] M. K. Choi, "Numerical calculation of light scattering from a layered sphere by the boundary-element method," Journal of the Optical Society of America A, vol. 18, no. 3, pp. 577-583, 2001.

[13] D. Chandler, Introduction to Modern Statitical Mechanics, Oxford University Press, New York, NY, USA, 1987.

[14] J. Wojcik, B. J. Robinson, D. A. Thompson, and P. Mascher, "Control of dielectric cap induced band-gap shift in $1.55 \mu \mathrm{m}$ laser structures," Journal of Vacuum Science and Technology A, vol. 20, no. 3, pp. 1076-1078, 2002.

[15] J. F. Hazell, D. A. Thompson, N. Bertsch, J. G. Simmons, B. J. Robinson, and G. I. Sproule, "Intermixing of InGaAsP/InGaAsP quantum-well structures using dielectric films," Semiconductor Science and Technology, vol. 16, no. 12, pp. 986-991, 2001.

[16] O. Hulko, D. A. Thompson, J. A. Czaban, and J. G. Simmons, "Quantitative analysis of compositional changes in InGaAs/InGaAsP quantum wells on GaAs induced by intermixing with a low temperature grown InGaP cap layer," Applied Physics Letters, vol. 89, no. 6, Article ID 061915, 2006.

[17] O. Hulko, D. A. Thompson, and J. G. Simmons, "Quantitative compositional analysis of quantum well intermixing using a low temperature MBE-grown InP cap layer," Physica Status Solidi C, vol. 3, no. 3, pp. 647-650, 2006.

[18] D. A. May-Arrioja, N. Bickel, A. Alejo-Molina, M. TorresCisneros, J. J. Sanchez-Mondragon, and P. LiKamWa, "Intermixing of InP-based multiple quantum wells for integrated optoelectronic devices," Microelectronics Journal, vol. 40, no. 3, pp. 574-576, 2009.

[19] Y. Wang, H. S. Djie, and B. S. Ooi, "Group-III intermixing in InAs/InGaAlAs quantum dots-in-well," Applied Physics Letters, vol. 88, no. 11, Article ID 111110, 2006. 


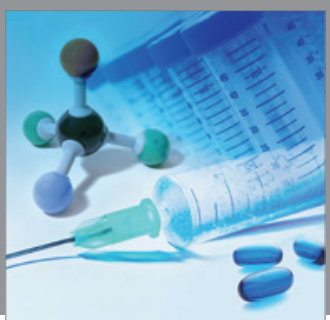

International Journal of

Medicinal Chemistry

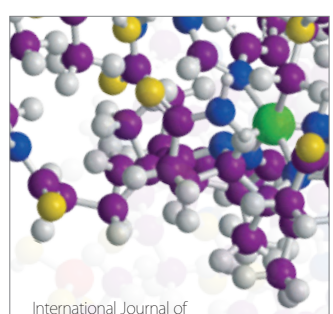

Carbohydrate Chemistry

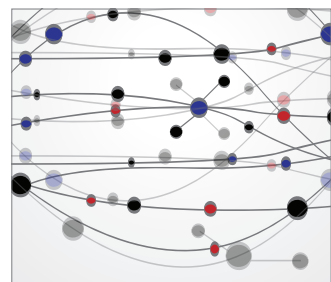

The Scientific World Journal
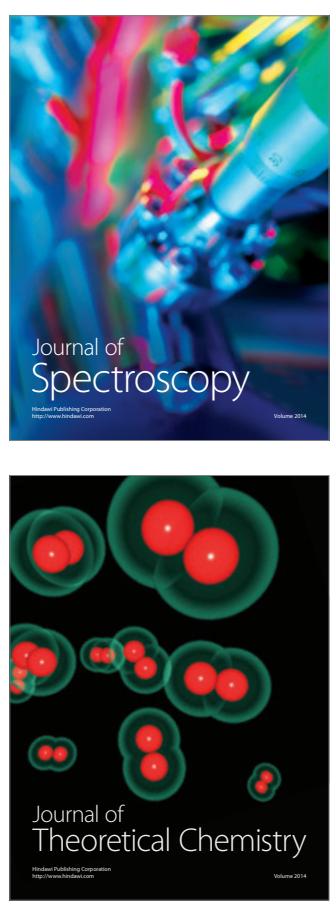
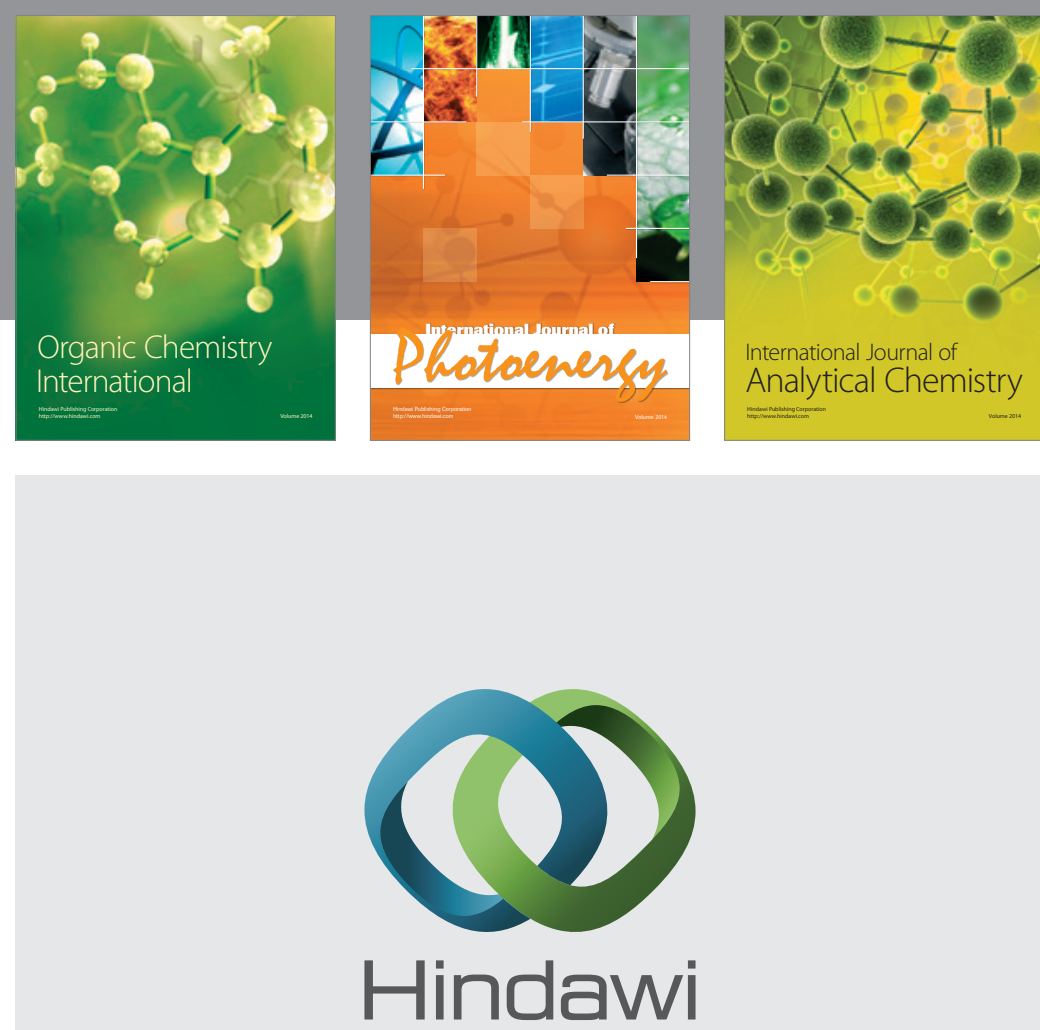

Submit your manuscripts at

http://www.hindawi.com
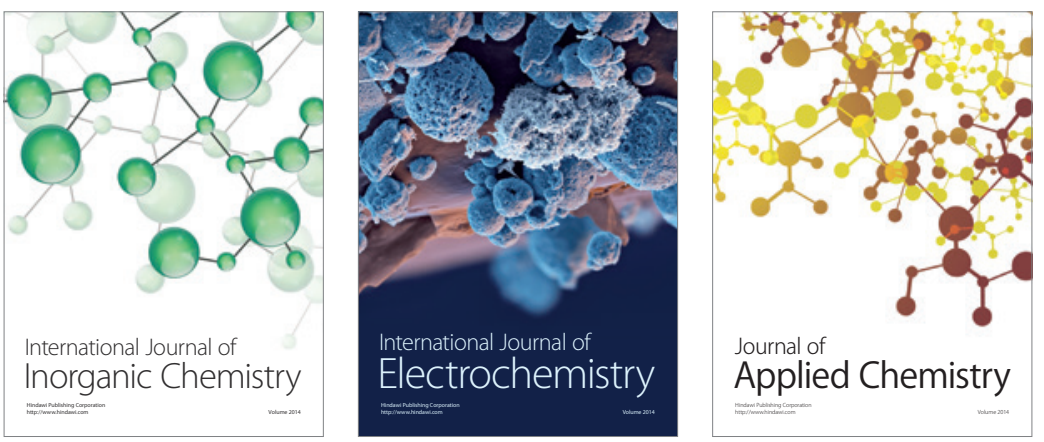

Journal of

Applied Chemistry
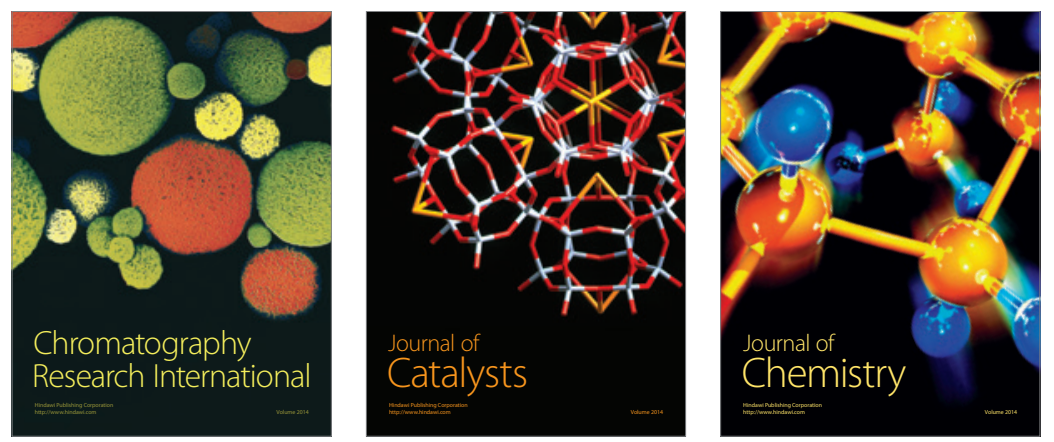
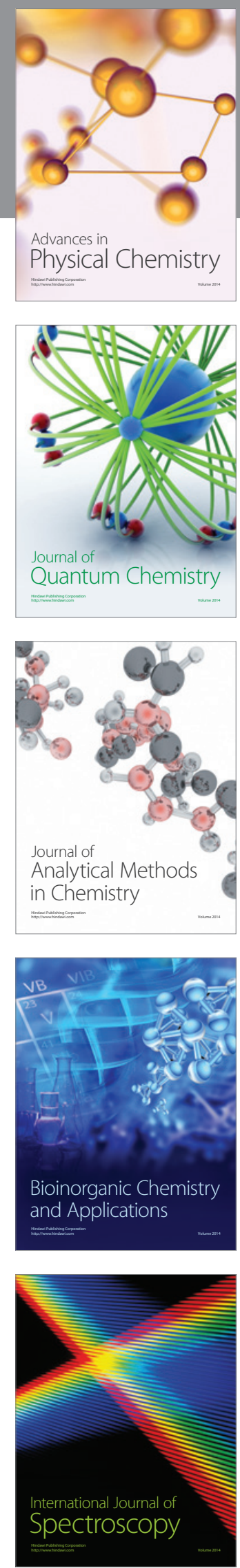